\title{
Translation and validation of the Child and the Adolescent HARDSHIP (Headache-attributed restriction, disability, social handicap and impaired participation) questionnaire into Danish language.
}

Jens Erik Jorgensen, Kate A McGirr, Hanne Oertved Korsgaard, Michael S Rathleff

Background: The prevalence of headaches among children and adolescents varies considerably between countries. This may be due to a lack of appropriate instruments to capture the prevalence. The purpose of this study was to translate the Child and Adolescent HARDSHIP Questionnaires from English into Danish language, conduct crosscultural adaptation, face validation by cognitive interviewing and conduct a pilot study exploring time requirements. Methods: The questionnaire was translated using the guidelines proposed by "The Global Campaign to Reduce the Burden of Headache". 25 children from 6 to 12 years of age completed the questionnaire with 24 hours between test and retest to assess reliability. 169 children and adolescents from 6 to 17 years of age completed the translated questionnaire to assess time requirements for completing it. Results: Only minor discrepancies were observed in the translation process. Test-retest reliability of the translated questionnaire showed substantial agreement (kappa: 0.65 to 0.78). The questionnaires were completed within 30 minutes (age 6 to 11 years of age) and within 15 minutes (age 12-17 years of age) respectively. Discussion: No major problems were observed in the forward translations of the questionnaires. The face validation prompted no major changes in the questionnaire. The face-to-face interviews showed that pupils of different ethnic backgrounds than Danish and pupils in the age group of 6 to 11 had more difficulty in understanding a minority of the questions. The Danish Child and Adolescent HARDSHIP questionnaire therefore complies with the intentions of the originators, aiming at a maximal completion time of 45 minutes and in comparison with actual completion time. The test-retest study showed substantial agreement between test and retest in the headache, migraine and $\mathrm{MOH}$ domains and questions referring to time. The Child and Adolescent HARDSHIP questionnaire, includes a section specifically recording a four-week period.The Child and Adolescent HARDSHIP questionnaire is intended to measure burden of headache in large populations and there is therefore no need to reflect the states of individuals. We therefore find the substantial reliability of the Danish version of the Child and Adolescent HARDSHIP questionnaire to be 
adequate, without supplementing with a diary. The pilot study indicates that headache is a major problem among children and adolescents in Denmark.95.3\% of the pupils have experienced headache in their life, $76.6 \%$ during the last year. $14 \%$ left school early because of their headache and $14.9 \%$ missed school during the last four weeks, due to headache.49.2\% have experienced headaches during the last week and $47 \%$ describe the headache as "quite bad". $24.1 \%$ have taken pills or medicine during the last week due to headache. This pilot study clearly demonstrates the need to investigate the burden of headache among Danish schoolchildren as it seems to have a profound effect on their lives. 
1 Translation and validation of the Child and the Adolescent HARDSHIP (Headache-attributed restriction,

2 disability, social handicap and impaired participation) questionnaire into Danish language.

3 Jorgensen $\mathrm{JE}^{1}$, McGirr K², Oertved HK , Rathleff $\mathrm{MS}^{2,3}$

4 Affiliations

5 1: Private Practice Clinic. Hjulmagervej 34, Aalborg

6 2: Department of Occupational Therapy and Physiotherapy, Aalborg University Hospital

7 3: Research Unit for General Practice in Aalborg and Department of Clinical Medicine, Aalborg University,

8 Denmark

\section{Corresponding author}

10 Jens Erik Jorgensen

11 Klinik for Fysioterapi, Hjulmagervej 34. 9000 Aalborg. Denmark

12 Phone: +4530239585 (mob)

13 Email: jeja26@gmail.com 


\section{Abstract \\ 15 Background}

16 The prevalence of headaches among children and adolescents varies considerably between countries.

17 This may be due to a lack of appropriate instruments to capture the prevalence. The purpose of this study

18 was to translate the Child and Adolescent HARDSHIP Questionnaires from English into Danish language,

19 conduct cross-cultural adaptation, face validation by cognitive interviewing and conduct a pilot study

20 exploring time requirements.

\section{Methods}

The questionnaire was translated using the guidelines proposed by "The Global Campaign to Reduce the Burden of Headache". 25 children from 6 to12 years of age completed the questionnaire with 24 hours between test and retest to assess reliability.169 children and adolescents from 6 to 17 years of age completed the translated questionnaire to assess time requirements for completing it.

\section{Results}

Only minor discrepancies were observed in the translation process. Test-retest reliability of the translated questionnaire showed substantial agreement (kappa: 0.65 to 0.78 ). The questionnaires were completed within 30 minutes (age 6 to11 years of age) and within 15 minutes (age 12 to 17 years of age)respectively.

\section{Discussion}

No major problems were observed in the forward translations of the questionnaires. The face validation prompted no major changes in the questionnaire.The face-to-face interviews showed that pupils of different ethnic backgrounds than Danish and pupils in the age group of 6 to 11 had more difficulty in understanding a minority of the questions. The Danish Child and Adolescent HARDSHIP questionnaire therefore complies with the intentions of the originators, aiming at a maximal completion time of 45 minutes and in comparison with actual completion time.

The test-retest study showed substantial agreement between test and retest in the headache, migraine and $\mathrm{MOH}$ domains and questions referring to time.

The Child and Adolescent HARDSHIP questionnaire, includes a section specifically recording a four-week period. The Child and Adolescent HARDSHIP questionnaire is intended to measure burden of headache in large populations and there is therefore no need to reflect the states of individuals. We therefore find the substantial reliability of the Danish version of the Child and Adolescent HARDSHIP questionnaire to be adequate, without supplementing with a diary.

The pilot study indicates that headache is a major problem among children and adolescents in Denmark. 
47 headache.49.2\% have experienced headaches during the last week and $47 \%$ describe the headache as 48 "quite bad". $24.1 \%$ have taken pills or medicine during the last week due to headache. This pilot study

49 clearly demonstrates the need to investigate the burden of headache among Danish schoolchildren as it 50 seems to have a profound effect on their lives. 
Introduction

52 The global burden of headache is a significant health concern. Headache disorders rank third among the 53 worldwide causes of disability, measured in years of life lost to disability (Steiner et al., 2015). In Europe,

54 the total annual financial cost of headache amongst adults aged 18 to 65 years is estimated to be $€ 173$

55 billion (Linde et al., 2012)).

56 The prevalence of headache in children and adolescents varies considerably. The prevalence of migraine 57 is between $3 \%$ and $11 \%$ while the prevalence of tension headache ranges from $10 \%$ to $24 \%$ " (Antonaci et 58 al. 2014). A review by Wöber-Bingöl et al., covering 64 studies on headache and migraine in children and adolescents reported a prevalence of $54.4 \%$ for any type of headache and $9.1 \%$ for migraine (Wöber-

60 Bingöl et al., 2013). Among 12 to 15 year old adolescents, Straube et al. found a prevalence of 66 to $71 \%$

61 for at least one episode of headache in the past three months, and 33 to $40 \%$ for weekly headaches

62 (Straube et al., 2013). This makes headache the most common neurological symptom and commonest

63 manifestation of pain in childhood, with a high risk of developing chronic manifestations into adulthood

64 (Antonaci et al., 2014). The wide range of prevalence may be the result of the different methods and

65 instruments used to capture the prevalence (Kernick et al., 2009).

The Global Campaign against Headache has launched initiatives to combat this issue and standardize the methods and instruments used in studies on headache. Patient reported outcome measures (PROMs) provide an objective measure of a subjective construct: that is, an individual's experiences and concerns in relation to their health, health care and quality of life (Haywood., 2007). The appropriate selection of an outcome measure should be guided by evidence of measurement properties, for example reliability, validity and responsiveness, and practical properties, such as patient acceptability and feasibility (Haywood., 2006). One of the requirements is a survey instrument with good cross-cultural validity

73 (Peters, Passchier., 2006)

The Global Campaign against Headache has recommended utilizing questionnaires such as the Child and Adolescent HARDSHIP Questionnaires in order to provide valid estimates of the prevalence and impact of headaches among children and adolescents. However, the Child and Adolescent HARDSHIP Questionnaires does not exist in Danish.

The purpose of the current study is to translate the Child and Adolescent HARDSHIP Questionnaires from English into the Danish language and conduct a cross-cultural adaptation to a Danish context including face validation by cognitive interviewing. The study also had the aim of completing a pilot study with a larger group of pupils within the target age group of 6 to 17 to test the time requirements for completing the questionnaires. 


\section{Materials and Methods}

\section{Ethical approval}

Before initiating the study, the authors contacted the Ethics Committee for North Jutland, Denmark. The Ethics committee stated that no approval was necessary, as the study did not include an intervention. Oral and informed consent was obtained from all participants. The study complied with the Declaration of Helsinki.

\section{The Child and Adolescent HARDSHIP questionnaire}

The Headache-Attributed Restriction, Disability, Social Handicap and Impaired Participation (HARDSHIP) questionnaire (Steiner et al., 2014) is designed for application by trained lay interviewers. HARDSHIP is a modular instrument incorporating demographic enquiry, diagnostic questions based on ICHD-3 beta criteria, and enquiries into components of headache-attributed burden. HARDSHIP has already demonstrated validity and acceptability in multiple languages and cultures (Steiner et al. 2014). A similar questionnaire has been developed to assess the global estimation of burden of headache in children and adolescents, The Child and Adolescent HARDSHIP Questionnaire (Wöber -Bingöl et al., 2014). It can be used to capture the prevalence of headache, the impact of headache on their lives and as a diagnostic tool to differentiate between tension type headache (TTH), migraine and Medication Overuse Headache $(\mathrm{MOH})$ type of headache. The Child and Adolescent HARDSHIP questionnaire consists of a total of 44 questions: one to record the date; two demographic questions; two screening questions for headache prevalence; 10 headache diagnostic questions (related to headache characteristics and associated symptoms); four questions enquiring into the frequency of headache and the use of abortive medication, four questions related to activity loss; three questions related to headache yesterday; six questions referring to other aspects of headache-attributed burden; 12 questions about quality of life. The questionnaire is acceptable, valid and feasible for a global assessment of the burden of headache in children and adolescents (Wöber -Bingöl et al., 2014).

\section{Translation procedure for the Child and Adolescent HARDSHIP questionnaire}


112 The translation procedure was done in accordance with the guidelines proposed by the Translation

113 Working Group for the translation of documents produced by Lifting The Burden (LTBT) (LTBT). The

114 process was supplemented with the guidelines produced by Beaton et al. (Beaton et al., 2000; Beaton et

115 al., 2007)), the principles of good practice proposed by ISPOR (Wild et al., 2005) and translating

116 instruments for cross-cultural studies in Headache Research (Peters and Passchier., 2006).

117 The procedure was as follows:

118 1) Permission to translate the Child and Adolescent HARDSHIP questionnaire. The

119 original questionnaires and translation guidelines were obtained from the developers by

120 e-mail. (Steiner et al.,2014; Wöbel-Bingôl et al.,2014)

121 2) Forward translation. Two independent bilingual Danish residents (T1 and T2), with

122 Danish as their birth languages, translated the questionnaires into Danish. T1 was

123 aware of the concepts being examined, whereas T2 was not (Beaton et al., 2000; Peters

124 and Passchier., 2006) A translation coordinator, overseeing but not carrying out the

125 translation, was selected according to the criteria stipulated by LTBT (LTBT).

126 3) Reconciliation. Separate meetings were held with each of the translators (T1 and T2)

127 to address discrepancies in the forward translations. A written report documented issues

128 in relation to the translation process (Beaton et al., 2000; Peters and Passchier., 2006) If

129 there were any unresolved queries, the original developers were contacted to resolve

130 the query (Steiner et al., 2014; Wöber-Bingöl et al., 2014).

131 4) The agreed-upon, forward-translated versions (T12) of the Danish questionnaire was

132 hereafter translated back to English by two Danish residents whose birth language was

133 English (BT1 and BT2). BT1 was a research physiotherapist, whereas BT2 had no

134 medical background. BT1 and BT2 were both blinded to the purpose of the

135 questionnaire and had not seen the original Child and Adolescent HARDSHIP

136 questionnaires (Beaton et al., 2000; Peters and Passchier., 2006).

137 5) The translated versions were compared to the original versions to ensure conceptual

138 equivalence; the remaining discrepancies and ambiguities were resolved among the

139 project manager, BT1, and BT2. The back-translation was forwarded to the original

140 author with a request to compare the original and back-translated versions and assess

141 their conceptual equivalence (LTBT).

142 6) Harmonisation. The harmonisation group consisted of the back- and forward 
143 translators, a research physiotherapist and a language competent physiotherapist. The

144 project manager communicated with each member of the harmonisation group

145 individually by email, due to geographic differences. Subsequently, through group-email

146 correspondence, the team agreed upon the harmonised versions of the Danish Child

147 and Adolescent HARDSHIP questionnaires. All members of the harmonisation group

148 approved the translated versions.

149 7) A test-retest study was conducted in a primary school. The ages of the participants 150 ranged from 6 to 12 years of age. The objective of the pilot study was to evaluate the

151 time needed for completion of the questionnaire within the different age groups and the

152 need for help and mediation of the participants by the teachers. The answers were

153 anonymous. The age group selected was in expectation of this being the age group

154 where additional help in understanding the questionnaire would be most needed.

155 8) A pilot study was conducted at a primary school. The ages of the participants ranged

156 from 6 to 17 years of age. The objective of the pilot study was to evaluate the time

157 needed for completion of the questionnaire within the different age groups and the need

158 for help and mediation of the participants by the teachers. The answers were

159 anonymous. The age group selected was in accordance with the target age of the

160 questionnaire.

$1619)$ Cognitive interviews were carried out at both schools. 12 children within the target

162 ages of 6 to 17, were interviewed while completing the questionnaire (LTBT). The

163 interviews varied between 30 to 45 minutes. The children were all affected by headache

164 and native speakers of the Danish language. The interviewer was a qualified teacher,

165 well-trained in the method of cognitive interviewing and proficient in interviewing

166 children. This procedure provided a validity check and the possibility to amend potential

167 errors and problems and to minimise future response errors and non-response (LTBT;

168 Kuusela and Paul., 2000 ; Wild et al. 2005)

169 10) The results from the cognitive interviews were reviewed and a final translated

170 version of each questionnaire was prepared. Any issues relating to the interpretation of

171 the questionnaires were documented in writing (LTBT; Kuusela and Paul., 2000 ; Wild et

172 al. 2005) 
173

174

175

176

177

178

179

180

11) A lay person with no medical or research background with good linguistic understanding (a school teacher) assessed the back-checked consensus-based translation for readability, grammatical correctness and cultural suitability. Queries were documented and sent to the co-ordinator (LTBT).

12) The final translated versions were proofread and checked for errors of spelling and grammar, then the layout was finalised by an expert committee consisting of the project manager, two research physiotherapists and a qualified teacher (LTBT; Beaton et al., 2000).

\section{3) A final report documenting the translation procedure was produced (LTBT).}

\section{Test-retest reliability of the Danish Child and Adolescent HARDSHIP questionnaire-DK}

The Child and Adolescent HARDSHIP questionnaire was completed twice with 24 hours between test and retest by 25 children aged 6 to 12 to assess test-retest reliability. The 24-hour period was chosen as headache due to practical reasons. The study sample was intentionally heterogeneous as the Child and Adolescent HARDSHIP questionnaire is intended for use in multiple conditions as suggested by the originator (Kuusela and Paul., 2000). The questionnaire was completed online using Google Docs within the school's intranet system. The response rate was $100 \%$ at both test and retest. See Table 1 for participant characteristics.

\section{Pilot study to assess the time requirements for completing the questionnaire.}

109 children age ranging from 6 to 11 years of age completed The Child HARDSHIP questionnaire and 60 children age ranging from 12 to 17 completed The Adolescent HARDSHIP questionnaire. The objective of the pilot study was to evaluate the time needed for completion of the questionnaire within the different age groups and the need for help and mediation of the participants by the teachers. The answers were anonymous. The age group selected was in accordance with the target age of the questionnaire. The pupils received the instruction of reading the questionnaire to answer the questions to the best of their ability. The teacher present clarified any queries as to the understanding of the questions.

\section{Cognitive interviews}

Cognitive interviews of 12 pupils with headache were performed to assess the harmonised versions in a local primary school. (LTBT., Beaton et al., 2007) A teacher, well trained in the method of cognitive interviewing, conducted the interviews. The pupils interviewed were within the target age groups of the questionnaire and randomly selected in six different school grades. (Table 1) The interview method 
employed was a "Thinking aloud" study (Someren et al., 1994) and varied between 10 to 35 minutes. During the course of a usability study, the children were asked to verbalise their thoughts and opinions while completing the questionnaire. This procedure provided a validity check and the possibility to amend potential errors and problems and to minimise future response errors and non-response (Kuusela and Paul., 2000).

\section{Statistics}

Demographics of the included pupils are given as mean and the standard deviation. Question 4 to 15 are answered on a categorical scale. Questions 16 to 22 are based on questions relating to the number of days and therefore numerical data. Question 23 consists of two categories, categorical and numerical data. Questions 27 numerical data. Questionlating to the number of days and therefore numerical data. Question 23 consists of two categories, categoric while completing the questionnaire. This procedure provirical data. The interpretations of kappa values follow Landis and Koch (Streiner,Norman,Cairny., 2014). The test-retest reliability of the total number of days was analysed using an Intraclass correlation (ICC 2.1).

\section{Results}

\section{Translation}

No major problems were observed in the forward translations of the questionnaires and only minor discrepancies were found by the originator, primarily consisting of differences in the choice of synonyms and use of prepositions. Examples are: the expression "official relations" in the original version has specific meaning which is not captured by "cooperation" in the translated version, the expression "exercise" in the original was not covered by the expression "movement" in the translated version, the original was "things" (i.e., some things), which is not the same as "anything" (i.e., all things). These discrepancies were corrected and approved by the originator (Kuusela and Paul., 2000).

\section{Cognitive interviews}

The interview with the pupils prompted no major changes in the questionnaire. Only a few minor changes were made (see supplementary web-appendix for a complete list). In the age group between 6 and 11 a problem was encountered relating to time 
230 recollection. The time span of 4 weeks and 1 year was difficult for the youngest. This 231 age group also showed difficulty in comprehending longer sentences. In the age group

23212 to 17 there were no difficulties, and the pupils assessed the questionnaire as

233 understandable.

\section{Pilot study}

235 Table 1 showing the characteristics of the included participants in the three studies

236 The pilot study included 169 pupils between 6 and 17 years of age. Completion time of the questionnaire

237 was between 3 and 30 minutes for the youngest and between 3 and 15 minutes for the oldest age group.

238 Pupils of different ethnic backgrounds had in general more difficulty in understanding a minority of the 239 questions. Pupils in the 6 to 11 age group, needed mediation to clarify a minority of the questions. The 240 pilot study indicates that headache is a major problem in these age groups. 95.3\% of the pupils have 241 experienced headache in their life, $76.6 \%$ during the last year. $49.2 \%$ have experienced headaches during 242 the last week, $47 \%$ describe the headache as quite bad. $24.1 \%$ have taken pills or medication during the 243 last week due to headache.14\% have left school early during as a result of their headache and $14.9 \%$ 244 missed school during the last four weeks, due to headache.

245 Reliability

246 "The test-retest study showed substantial agreement between test and retest in the headache, migraine 247 and $\mathrm{MOH}$ domains (absolute agreement $=76.9$ to $96.2 \%$ and kappa $=0.65$ to 0.78 ). The sections of the 248 questionnaire referring to time, indicated almost perfect agreement. ICC 0.85 (95\% CI: 0.67, 0.93)" 249 Question 23 showed almost perfect agreement (absolute agreement $95.7 \%$ and kappa = 0.78) (Table 2)

Table 2 Test-retest values of the HARDSHIP questionnaires.

\section{Discussion}

252

An internationally recommended translation procedure was used to produce a Danish version of the Child and Adolescent HARDSHIP questionnaires, and a rigorous translation methodology was used to ensure the questionnaires were translated and adapted into a Danish context. Cognitive interviews were used to provide a face validation of the translated versions of the Child and Adolescent HARDSHIP questionnaire, thereby minimizing non-responses and response errors (LTBT; Wild et al., 2005).

No major problems were observed in the forward translations of the questionnaires. The face validation prompted no major changes in the questionnaire. The face-to-face interviews showed that pupils of different ethnic backgrounds than Danish and pupils in the age group of 6-11 had more difficulty in understanding a minority of the questions. 
The Danish versions were completed within 30 minutes for the age group of 6 to 11, and within 15 minutes for the age group of 12 to17. In the original questionnaire (Wöbel-Bingöl et al. 2014) the average time needed to complete the questionnaire was within 9 minutes (range 5 to 39 minutes), with a target of maximum 45 minutes. The Danish Child and Adolescent HARDSHIP questionnaire therefore complies with the intentions of the originators, aiming at a maximal completion time of 45 minutes and in comparison with actual completion time.

The test-retest study showed substantial agreement between test and retest in the headache, migraine and $\mathrm{MOH}$ domains and questions referring to time.

The 24 hours between test and retest was chosen due to practical issues at the schools involved. A testretest period of between two days and two weeks has been recommended (Nunnally and Bernstein., 1994). Shorter periods may be associated with pupils enabling the recollection of answers, thereby inflating reliability (Nunnally and Bernstein., 1994). The accuracy of children's ability to recall pain intensities has been shown to be high with little decrement over a 1-week period (Zonnefeld et al., 1997) and a study by van den Brink and colleagues (Van den Brink, Bandell-Hoekstra, Abu-Saad., 2001) investigating the recall bias in paediatric headache, showed that recall errors occur when children are asked to report their headaches in a retrospective questionnaire. However, unreliability in frequency estimates is of particular concern among individuals with high-frequency (chronic) headache. (Houle et al., 2013) Employing shorter recall intervals when assessing headache frequency may improve accuracy (Houle et al., 2013)

By reducing the test-retest interval the possible fluctuation of headache in children, where headaches may occur within weekly intervals and varying duration (Rho et al., 2012; Straube et al., 2013), may also influence reliability if the time interval was to be expanded by too large a time interval. We do not expect the relative short period of 24 hours to be disadvantageous, as it may show a higher accuracy and recall bias may be eliminated. The Child and Adolescent HARDSHIP questionnaire (Wöber-Bingöl et al., 2014) has a total of 44 questions, which in itself may make recalling answers to specific questions difficult.

The Child and Adolescent HARDSHIP questionnaire, includes a section specifically recording a four week period. In this study, absolute agreement levels had a tendency to diminish as the recall time span lengthens. This is expected as recall bias increases over time (Stewart et al., 2000; Van den Brink, Bandell-Hoekstra, Abu-Saad., 2001; Zwart et al., 2004). The Child and Adolescent HARDSHIP questionnaire is intended to measure burden of headache in large populations and there is therefore no need to reflect the states of individuals. We therefore find the substantial reliability of the Danish version of the Child and Adolescent HARDSHIP questionnaire to be adequate, without supplementing with a diary (Zwart et al., 2004; Wöber-Bingöl et al., 2014,).

The pilot study indicates that headache is a major problem among children and adolescents in Denmark. See table 3 . This pilot study clearly demonstrates the need to investigate the burden of headache among 
Danish schoolchildren as it seems to have a profound effect on their lives. The pilot study included a small sample size and therefore, validity of the results must be interpreted with care.

Table 3: Impact of headache on pupils in pilot study

\section{Perspectives and future research}

Publishing the translation of a health measurement instrument is important in order to avoid the emergence of multiple versions of the instrument and to demonstrate that the translation procedure was rigorous. A translated questionnaire also makes it possible to compare findings between and within countries (Zwart et al., 2004). The translation of the Child and Adolescent HARDSHIP Questionnaire to Danish versions was performed using rigorous methodology, adapted culturally, and face validated to a Danish context.

Previous work suggests that the Child and Adolescent HARDSHIP Questionnaire predicts substantial impact of headache in the respective age groups of 6 to 11 and 12 to 17 years of age. (Wöber-Bingöl et al., 2014). The Child and Adolescent HARDSHIP questionnaire may therefore identify the prevalence of headache in Danish youth and help distinguish the types of headache they suffer from.

\section{Conclusion}

The translation of the Child and Adolescent HARDSHIP Questionnaire to Danish versions showed substantial test-retest study. The Child and Adolescent HARDSHIP Questionnaire may be used in a Danish context to identify the prevalence and impact of different types of headaches among children and adolescents.

\section{Article highlights:}

- The reliability of the Danish Child and Adolescent HARDSHIP questionnaires showed substantial agreement

- The sections of the questionnaire referring to time, showed substantial agreement

- The pilot study indicates that headache may be a major problem in the age groups of 6 to 11 and 12 to 17 years of age in Denmark. 


\section{Acknowledgements}

323 The authors thank Prof.Dr. Timothy J Steiner, Department of Neuroscience, Norwegian

324 University of Science and Technology, Trondheim, Norway ${ }^{(1)}$ Department of

325 Neuroscience, Imperial College London, London, UK ${ }^{(2)}$ (t.steiner@imperial.ac.uk) for

326 kindly permitting the translation of the original of the Headache-Attributed Restriction,

327 Disability, Social Handicap and Impaired Participation (HARDSHIP) Child and

328 Adolescent questionnaire and for advice given during the translation process.

329 The authors acknowledge the help received in the translation process of Prof. Dr. Çiçek

330 Wöber-Bingöl, Department of Child and Adolescent Psychiatry, Medical University of

331 Vienna, Währinger Gürtel 18-20, 1090 Vienna, Austria. (yasar.woeber-

332 bingoel@meduniwien.ac.at) We also thank the members of the Harmonisation

333 Committee for their important contribution to the translation procedure.

334 The authors express their gratitude to participating students and all staff of the participating schools for

335 their excellent cooperation and contribution to this study 


\section{References:}

1. Antonaci F, Voiticovschi-losob C, Di Stefano AL, Galli F, Ozge A. Balottin U. The evolution of headache from childhood to adulthood: a review of the literature. J Headache Pain 2014; 15:15

2. Beaton DE, Bombadier C, Guilleman F, Ferraz MB. Guidelines for the process of cultural crossadaption of self-reported measures. Spine 2000: 25(24): 3186-3191

3. Beaton DE, Bombadier C, Guilleman F, Ferraz MB. Recommendations for the cross-cultural adaption of the DASH and QuickDASH outcome measures. Institute for Work of Health 2007

4. Haywood KL. Patient-reported outcome I: Measuring what matters in musculoskeletal care. Musculoskelet. Care.2006; 4(4):187-203

5. Haywood KL. Patient-reported outcome II: selecting appropriate measures for musculoskeletal care. Musculoskelet. Care. 2007;5(2): 72-90

6. Houle T T, PhD, Turner D P, Houle T A, Smitherman T A, Martin V, Penzien D B, Richard B. Lipton R B. Rounding Behavior in the Reporting of Headache Frequency Complicates Headache Chronification Research. Headache. 2013 Jun; 53(6): 908-919.

7. Kernick D, Reinhold D, Campbell JL Impact of headache on young people in a school population. Br J Gen Pract. 2009;Sep.59(566):678-81

8. Kuusela, H,Paul, P. "A comparison of concurrent and retrospective verbal protocol analysis". American Journal of Psychology (University of Illinois Press).2000;113 (3): 387-404

9. Landis JR and Koch GC. The measurement of observer agreement for categorical data. Biometrics 1977; 33:159-174.

10. Linde M, Gustavsson A, Stovner LJ, Steiner TJ, Barré J, Katsarava Z, Lainez JM, Lampl C, Lantéri-Minet M, Rastenyte D, Ruiz de la Torre E, Tassorelli C, Andrée C. The cost of headache disorders in Europe: the Eurolight project. Eur J Neurol. 2012; May;19(5):703-11

11. LTBT. Lifting The Burden Translation Working Group (LTBT) http://www.l-tb.org/index.cfm/spKey/horizontal_activities.supporting.linguistic_translation.html (accessed May and September 2015)

12. Nunnally JC and Bernstein IH, Psychometric Theory. Third Edition. 1994 Chapter 7. ISBN C-C7047849-X

13. Peters M, Passchier J. Translating Instruments for Cross-Cultural Studies in Headache Research. Headache 2006;46:82-91 
366

367

368

369

370

371

372

373

374

375

376

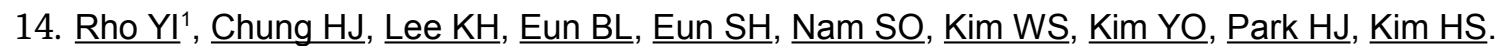
Prevalence and clinical characteristics of primary headaches among school children in South Korea: a nationwide survey. Headache. 2012 Apr;52(4):592-9.

15. Someren MW, Barnard YF, Sandberg JAC, The Think Aloud Method .A practical guide to modelling cognitive processes. Department of Social Science Informatics University of Amsterdam , Published by Academic Press, London, 1994; ISBN 0-12-714270-3

16. Steiner TJ, Gururaj G, Andrée C, Katsarava Z, Ayzenberg I, Yu S-Y, Jumah MA, Tekle-Haimanot R, Birbeck GL, Herekar, A, Linde M, Mbewe E, Manandhar K, Risal A, Jensen R, Queiroz LP, Scher Al, Wang S-J,Stovner LJ. Diagnosis, prevalence estimation and burden measurement in population surveys of headache: presenting J Headache Pain 2014; 15:3.

17. Steiner TJ, Birbeck GL, Jensen RH, Katsarava Z, Stovner LJ, Martelletti P. Headache disorders are third cause of disability worldwide. J Headache Pain. 2015; 16:58

18. Stewart WF, Lipton RB, Kolodner KB, Sawyer J, Lee C, Liberman JN. Validity of the Migraine Disability Assessment (MIDAS) score in comparison to a diary-based measure in a population sample of migraine sufferers. Pain. 2000;88(1):41-52

19. Straube A, Heinen F, Ebinger F, von Kries R. Headache in school children: prevalence and risk factors. Dtsch Arztebl Int. 2013 Nov 29;110(48):811-8

20. Streiner D, Norman G. Cairney J. Health Measurement Scales: A Practical Guide to Their Development and Use. Oxford: Oxford University Press. 2014. Chapter 8.ISBN-13: 9780199685219

21. Van den Brink, Bandell-Hoekstra EN, Adu-Saad HH. The occurrence og recall bias in pediatric headache: a comparison og questionnaire and diary data.Headache.2001;Jan:41(1):11-20

22. Wild D, Grove A, Martin,M, Eremenco S, McElroy V, Verjee-Lorenz, ISPOR Task Force for translation and Cultural Adaption (2005). Principles of good practice for the translation and cultural adaption process for patient-reported outcomes (PRO) measures: Report of the ISPOR task force for translation and cultural adaption. Value in Health. The Journal of the International Society for Pharmacoeconomics and Outcomes research 2005; 8(2): 94-104

23. Wöber-Bingöl C. Epidemiology of migraine and headache in children and adolescents. Curr Pain Headache Rep.2013; 17:341

24. Wöber-Bingöl C, Wöber C, Uluduz D, Uygunoğlu U, Aslan TS, Kernmayer M, ZeschH-E, Gerges N T A, Wagner G, Siva A, Steiner TJ. The global burden of headache in children and adolescents - developing a questionnaire and methodology for a global study. J Headache Pain 2014;15:86. 
398

399

400

401

402

25. Zonneveld LN, McGrath PJ, Reid GJ, Sorbi MJ . Accuracy of children's pain memories. Pain. 1997; Jul;71(3):297-302.

26. Zwart J-A, Dyb.G, Holmen TL, Stovner,LJ, Sand,T. .The prevalence of migraine and tension-type headaches among adolescents in Norway. The Nord-Trøndelag Health Study (Head- HUNTYouth), a large population-based epidemiological study. Cephalalgia, 2004; 24; 373-379 


\section{Table 1 (on next page)}

Basic characteristics of the pupils included in the study.

Table 1: Basic characteristics of the pupils included in the study. 


\begin{tabular}{|c|c|c|c|}
\hline \multicolumn{4}{|c|}{ Basic Pupil Characteristics } \\
\hline & Pilot Study & Think aloud & Test - retest \\
\hline Pupils included (n) & 169 & 12 & 26 \\
\hline Gender & & & \\
Fale (\%) & $82(48.5 \%)$ & $5(41.7 \%)$ & $12(46.2 \%)$ \\
& $87(51.5 \%)$ & $7(58.3 \%)$ & $14(53.8 \%)$ \\
\hline Mean age ( \pm SD) & $11.2( \pm 2.5)$ & $7.6( \pm 1.2)$ & $10.7( \pm 1.9)$ \\
& & & \\
\hline
\end{tabular}

2 Table 1: Basic characteristics of the pupils included in the study. 


\section{Table 2 (on next page)}

Test-retest values of the HARDSHIP questionnaire.

Table 2 showing the test-retest values of the HARDSHIP questionnaire. 


\begin{tabular}{|c|c|c|c|}
\hline \multicolumn{4}{|c|}{$\begin{array}{l}\text { Test-retest study. Kappa, agreement percentage and Intraclass Correlation } \\
\text { Coefficient (ICC) }\end{array}$} \\
\hline $\begin{array}{l}\text { Domains } \\
\text { Questions }\end{array}$ & $\begin{array}{l}\text { Absolute } \\
\text { Agreement } \\
\quad \%\end{array}$ & Kappa & $\begin{array}{l}\text { Intraclass Correlation } \\
\text { Coefficient (ICC) }\end{array}$ \\
\hline $\begin{array}{l}\text { Screening } \\
\text { Q4-5 }\end{array}$ & 96.2 & 0.78 & \\
\hline $\begin{array}{l}\text { Diagnostic } \\
\text { Q6-15 }\end{array}$ & 86.8 & 0.77 & \\
\hline $\begin{array}{l}\text { Impact headache on life the past } 2 \text { and } 4 \\
\text { weeks } \\
\text { Q } 16-22\end{array}$ & & & 0.85 \\
\hline $\begin{array}{l}\text { Impact parents: loss of work due to child's } \\
\text { headache } \\
\text { Q } 23\end{array}$ & 95.7 & 0.78 & \\
\hline $\begin{array}{l}\text { Impact Yesterday } \\
\qquad \text { Q 24-26 }\end{array}$ & 86.5 & 0.70 & \\
\hline $\begin{array}{l}\text { Impact last } 4 \text { weeks } \\
\text { Q 27-32 }\end{array}$ & 82.5 & 0.73 & \\
\hline $\begin{array}{l}\text { Quality of life in general } \\
\qquad \text { Q 33-44 }\end{array}$ & 78.9 & 0.70 & \\
\hline
\end{tabular}


2 Table 2 Test-retest values of the HARDSHIP questionnaire. 


\section{Table 3 (on next page)}

Table_3._Impact_of_headache_on_pupils_in_pilot_study 


\begin{tabular}{|c|c|}
\hline \multicolumn{2}{|c|}{ Pupils having experienced headache in pilot study (n=169) } \\
\hline Experienced headache in their life & $161(95.3 \%)$ \\
\hline $\begin{array}{r}\text { Experienced headache during the } \\
\text { last week }\end{array}$ & $129(76.6 \%)$ \\
\hline Experienced headache during the & $83(49.2 \%)$ \\
\hline Describe headache as quite bad & $79(47 \%)$ \\
\hline Have taken pills or medication during \\
the last week
\end{tabular}

1

2 Table 3: Impact of headache on pupils in pilot study 